\title{
FEEDER LOAD COMPOSITION TRACKING FOR SMART METERED LOW VOLTAGE CIRCUITS
}

\author{
Bruce STEPHEN \\ University of Strathclyde - UK \\ bruce.stephen@strath.ac.uk
}

\author{
Stuart GALLOWAY \\ University of Strathclyde - UK \\ stuart.galloway@strath.ac.uk
}

\begin{abstract}
As Distributed Generation penetrates Low Voltage networks in greater quantities, the behaviour of the loads on these circuits, typically small and residential customers, must be better understood to avoid unnecessary investment and capitalize on increased efficiencies. Estimating thermal constraints as well as islanding capabilities hinges on accurate and representative load profiling, which requires periods of typical behaviour to be gathered through metering. The increasing availability of Smart Meters offers a solution to this with high frequency load measurements that align with generation dispatch periods. Prior work has taken steps to develop finer grained load profiles than those developed at the national level but given the high propensity for variability in residential customers, such metrics are overly general for small power systems. This paper takes previous work on load profiling at MV and LV levels and uses it to generate load profile compositions for learning the composition of customers that make up an LV feeder load and how it evolves over time. A simplification of a residential load profile model is applied to a set of real AMI data on a simulated feeder, resulting in 3 categories of user, a stratification learned from historical metering data. This yields an abstracted disaggregation of the loads on the feeder that accommodates the high variability and the heterogeneous profiles within the residential loads. Since compositional data is defined over the simplex rather than a real space, this restricts the statistical tools available for modelling to an inflexible subset. The solution presented circumvents this problem by utilising transforms that map the contributions to the aggregated feeder load into real space permitting a wider selection of analysis tools to be applied. A demonstration using a year's worth of smart meter data is provided to show how latent traits in load composition and forecasted changes can be tracked over time using a modified linear dynamical system. This technique can be used in system planning to anticipate reversed power flows on $L V$ networks where demand is insufficient to absorb distributed generation this avoiding the need for expensive tap changer upgrades or up rating the thermal limit on the lines.
\end{abstract}

\section{INTRODUCTION}

From a Distribution Network Operators perspective, understanding loads at the residential level facilitates realisation of a number of key Smart Grid technologies. Integration of renewable generation, scheduling of localised storage and provision of ancillary services require accurate load profiling. Aside from this, understanding the true nature of load can allow capacity planning and deferment of network investment. Although large customers attached to the MV network have been metered for several years, the adoption of higher resolution metering for residential customers through the deployment of Smart Metering or Advanced Metering Infrastructure (AMI) opens up new questions in terms of understanding their loads. Industrial customers have been noted to be predictable [1] as they undertake similar tasks regularly. Domestic loads can vary considerably from day to day and between identical properties [2]; much of this variability stems from the variability of domestic routine and the appliances in the home. Electric space and water heating requirements of overall metered residential loads will be subject to seasonal influence as will the use of certain appliances such as clothes dryers. Exactly how this evolves and how the evolution varies across dwellings is not clear. This paper proposes a means of modelling the behaviour of the residential loads that are attached to an LV network by grouping these and then monitoring how the membership of groups changes over time.

\section{NETWORK MODEL}

A residential area is modelled using the IEEE 123 node test feeder [3] with all plant, topology and constraint assumptions being retained. The network diagram for this is show in figure 1 . The 114 spot loads are populated with anonymised 30 minute meter advances obtained from a UK residential area. For the purposes of testing on a changing season, the network model is run from January to July. No embedded generation is considered and network loads are assumed to be balanced. 


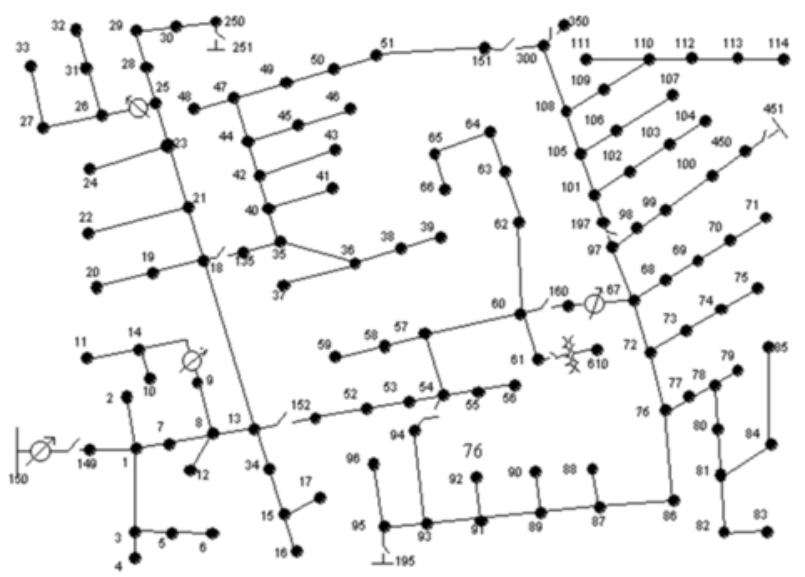

Figure 1. IEEE 123-Node Test Feeder [3].

The meteorological conditions for the area are also utilised to add context; for the purposes of this paper, this amounts to maximum ambient temperature.

\section{STRATIFYING LOADS}

Stratification of customer advance data has been identified as one possible way of segmenting residential customers into particular usage classes $[3,4,5]$. Different space and water heating technologies coupled with building fabric and utilization will lead to different quantities of energy usage in the domestic sector. Domestic routine will shape the size and position of peaks and the variability thereof. Various strategies for dividing residential loads into its constituent subpopulations have been proposed. [4] divided profiles into 48 half hourly periods and stratified the magnitude of loads in each period. [5] discriminated on shape and magnitude of profile using various clustering techniques. This is a prerequisite stage to identifying how a particular section of network is loaded and who the dominant

contributors are.
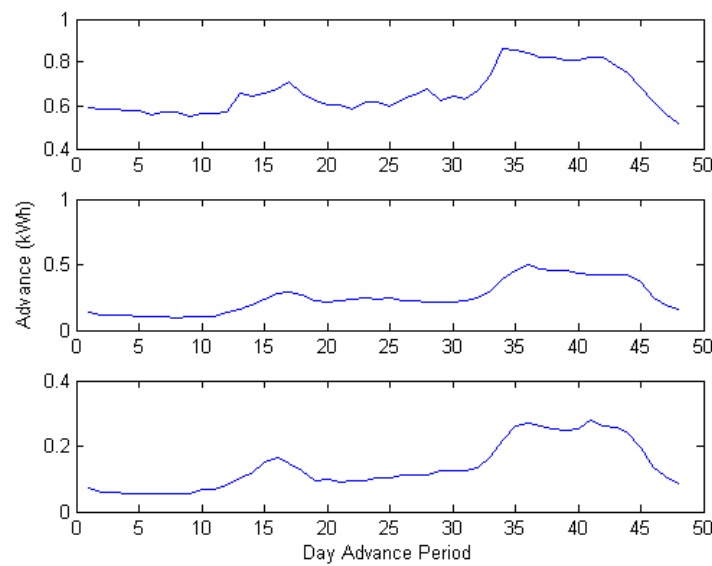

Figure 2. 3 Profile stratification of 1 months worth of residential loads: (from top) high usage, medium usage, low usage.
Using the clustering techniques proposed in [5], the 3 mean profiles shown in figure 2 are recovered. These are ordered from high to low according to their overall daily usage. What this does for a DNO is abstracts loads into 'big' and 'small' customers on a residential feeder. On a given day a customer on the network will exhibit a profile that is closest to at most one of these mean profiles, allowing them to be labeled accordingly.

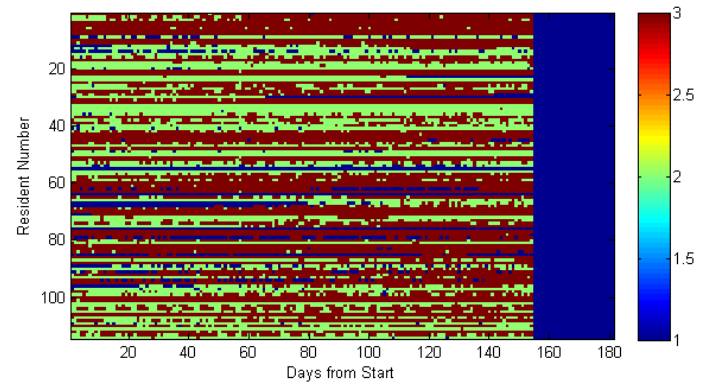

Figure 3. Profile class assignment over 150 days using the stratification model shown in figure 2 .

Figure 3 shows how the 114 customers on the network were labelled over the course of the 150 days they were monitored: most were low (red) or medium (green) usage, but there are small amounts of high (blue) usage customers appearing sporadically. What would be useful, would be to track what proportion are in each usage group on the network and how they move between groups.

\section{COMPOSITIONAL LOAD PROFILING}

Stratification of loads on an LV network will result in a composition of loads from various strata making up the aggregated load at the feeder transformer. Compositional data [6] formally exists in the Simplex: each point in the coordinate system sums to a constant. Figure 4 shows the proportions of loads in each of the 3 groups on the feeder.

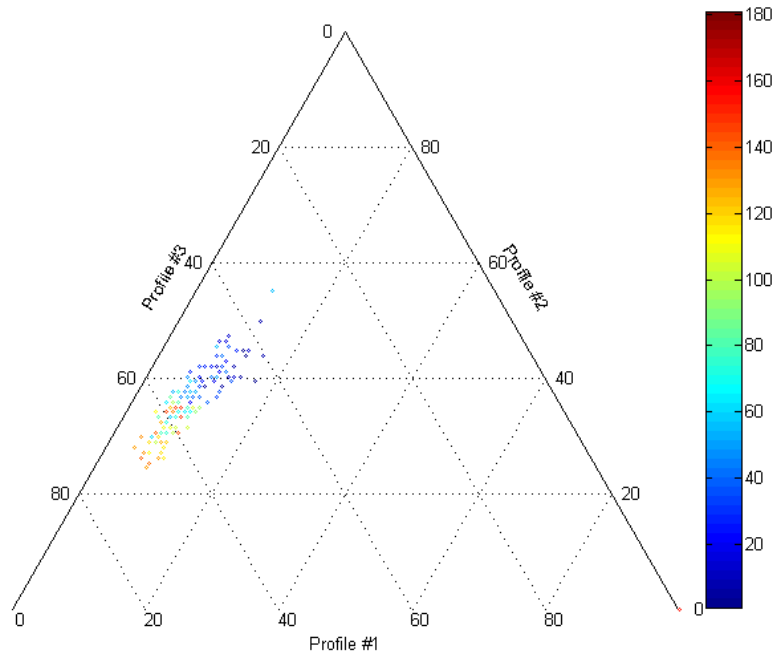

Figure 4. Composition of profile classes over the time period shown in figure 3 plotted in a 3 -simplex.

The colour of the points in figure 4 represents the number of 
days from the start - the movement of the residential loads from a dominant proportion of medium sized loads to a dominant proportion of small loads as the trial moves to the spring and summer months. Although the Barycentric coordinate system for representing the Simplex makes visualisation easier, it presents a problem for employing analytics which have an inherent assumption of Euclidean distance. This stems from dependence between dimensions in the simplex caused by their common denominator enforcing the constant sum constraint. [6] proposed the use of a transformation to data in the Simplex to map it into a Real space thus allowing a greater choice of analytics to be used. The log-ratio transform is as follows:

$$
x_{i}=\log \left(\frac{z_{i}}{z_{D}}\right), i \neq D
$$

This maps the transforms the compositional variable $Z$ of dimension $\mathrm{D}$, into the variable $\mathrm{X}$, of dimension D-1, in Real space. As noted in [6], a multivariate Gaussian distribution could then be used to represent compositional data and to provide a superior fit to distributions over the Simplex such as the Dirichlet which had limited flexibility in the dependency structures it could express and is computationally more difficult to deal with. Figure 5 shows the composition mapped into 2 dimensional real space.

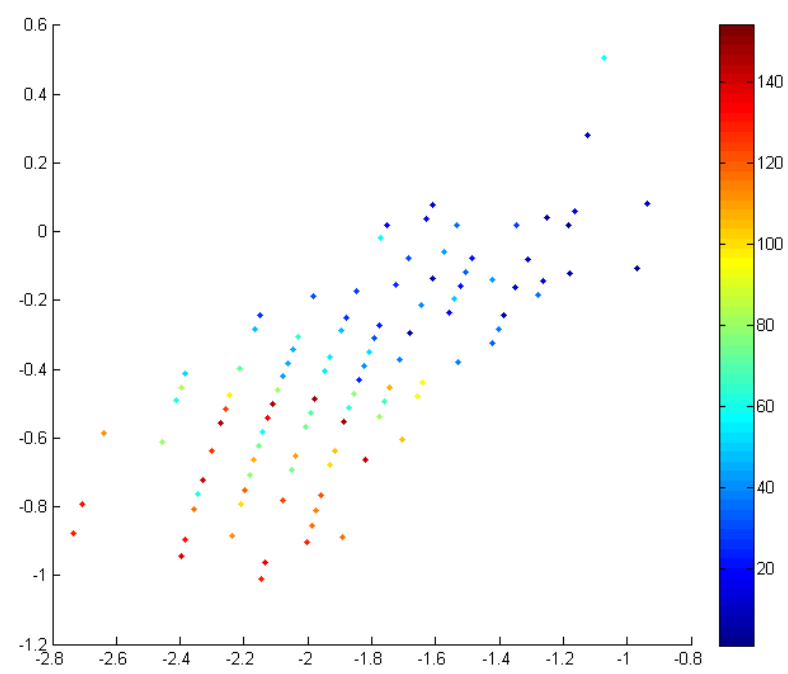

Figure 5. Log ratio transformation of load composition in Real space representation.

Again, the colour axis in the plot represents the time in days from the start of the trial, with the evolution of behaviour. Note that the axes in the transformed space no longer correspond to the composition proportions, although context can be recovered by performing the inverse transform.

\section{TRACKING GROUP BEHAVIOUR}

Seasonal change, or more specifically meteorological change, will invoke changes in longer period energy usage behaviour. The composition of stratified loads has reduced the 114 by 48 dimensional time series into a 2 dimensional one, with much of the daily variability noise accommodated by the stratification process. The network behaviour over time can be tracked as the movement of a point in the two dimensional space as shown in figure 5.

\section{Kalman Filter}

The Kalman Filter [7] is a linear Gaussian model commonly used in spatial tracking applications such as radar and image processing. The Kalman Filter permits continuous estimation of a non-stationary Gaussian distribution in a sequentially updated manner, i.e. there is no need for offline learning. Its parameterisation yields a smoothed estimate of this mean as well as a likelihood measure of how the predicted and actual observations compare. The Kalman filter mean can be transformed back into the simplex to show how the composition of loads changes over time. This allows it to detect anomalous conditions, volatility, change points and forecasting to be carried out. The key problem is that compositional data is not Gaussian distributed; however, employing a Kalman Filter in the Log Ratio space allows changes in composition to be tracked in an online manner in a way that would have been intractable or suboptimal in the Simplex.

\section{Time Evolution of Group Behaviour}

The Kalman filter tracks mean $\mu$ and covariance $\sum$ of a multivariate Gaussian distribution. The Gaussian distribution represents the observation likelihood with respect to the model parameters learned from past observations:

$$
P\left(x_{t} \mid \mu_{t}\right)=N\left(x_{t} ; \mu_{t}, \Sigma_{t}\right)
$$

Figure 6 shows the trace of the $\log$ of the observation likelihood from the Kalman Filter.

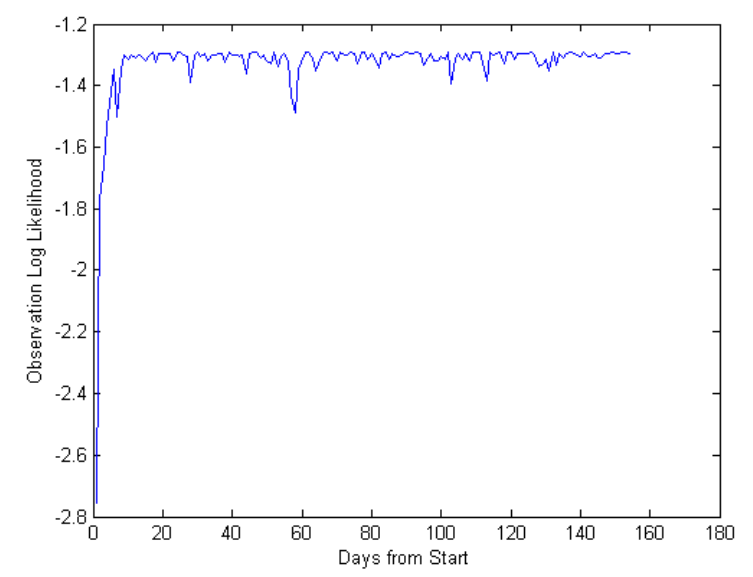

Figure 6. Filter observation log likelihood drops with abrupt changes in group behaviour.

Drops in log likelihood represent observations that were not 
expected by the model based on the observations it had previously received i.e. abrupt changes. Notable events occur around days 10, 60 and between 100 and 120 of the trial period. To understand the context of this, the temperature is given in figure 7.

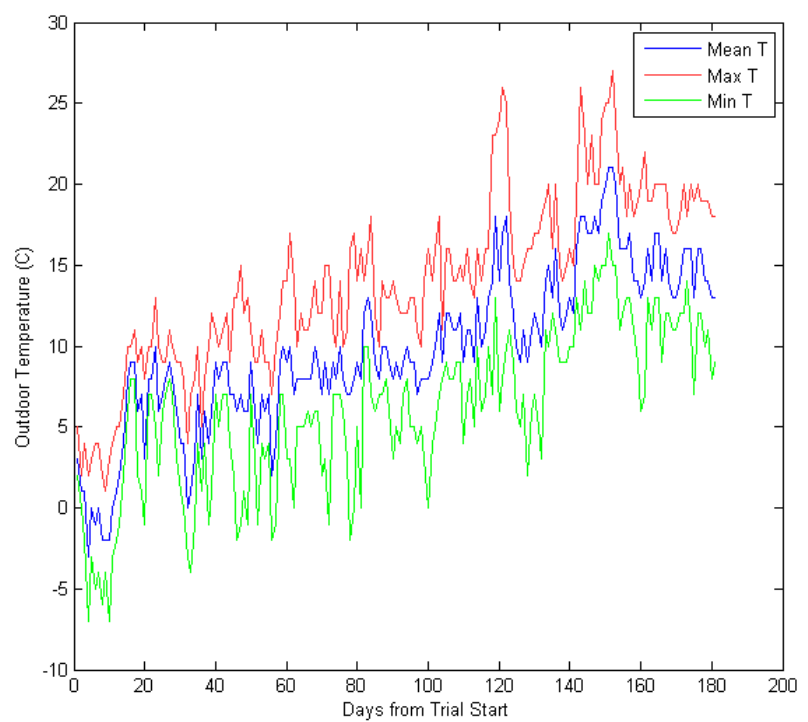

Figure 7. Daily temperatures over the monitoring period.

Day 10 has a significant drop in minimum temperature; day 60 has a sudden increase in maximum temperature and between days 100 and 120 there are significant downward excursions for minimum temperature followed by upward excursions for maximum temperature. All of these would influence electric heating usage patterns hence the abrupt change detected by the filter.

\section{FUTURE WORK}

This paper has presented a means for tracking changes to the make up of loads on residential feeders. This has been shown working on a day by day basis and has allowed anomalous conditions or abrupt changes in behaviour of the loads to be detected. A relatively simple model was used as the basis for this which relied heavily on linear relations between daily behaviour. This model showed how the existence and potential occurrence of various sizes of residential load could be tracked. This in turn can advise on the potential for breaching plant and thermal limits. In future work, it is expected that more sophisticated models will be developed that will allow non-linear relations or multiple types of relations [8] to be accommodated. Such models could allow DNOs to forecast or extrapolate from current to future load composition scenarios and better plan their network investment for greater reliability.

\section{ACKNOWLEDGEMENTS}

This work was undertaken as part of the ORIGIN project. The research leading to these results has received funding from the European Union Seventh Framework Programme
(FP7/2007-2013) under grant agreement $\mathrm{n}^{\circ} 314742$.

\section{REFERENCES}

[1] A. Mutanen, M. Ruska, S. Repo \& P. Jarventausta, 2011, "Customer Classification and Load Profiling Method for Distribution Systems", IEEE Trans. Power Delivery. vol. 26, no. 3, 1755-1763.

[2] K. Gram-Hanssen, 2009, "Standby consumption in households analysed with a practice theory approach ", Journal of Industrial Ecology, vol. 14, no. 1.

[3] IEEE PES - Power System Analysis, Computing and Economics Com., Distribution System Analysis Subcommittee "IEEE 123 Node Test Feeder", 2004

[4] B. Stephen \& S. Galloway, 2012, "Domestic Load Characterisation through Smart Meter Advance Stratification", IEEE Trans. Smart Grid, vol. 3, no. 3, 1571-1572.

[5] B. Stephen, A. Mutanen, S. Galloway, G. Burt \& P. Jarventausta, 2013, "Enhanced Load Profiling for Residential Network Customers", IEEE Trans. Power Delivery, in Review.

[6] J. Aitchison, 1982, "The Statistical Analysis of Compositional Data", Journal of the Royal Statistical Society. Series B (Methodological), vol. 44, no. 2, 139177.

[7] R.E. Kalman, 1960, "A New Approach to Linear Filtering and Prediction Problems", Trans. ASME-Journal of Basic Engineering. vol. 82, srs. D, 35-45.

[8] Kevin Murphy. Learning Switching Kalman Filter Models. Compaq Cambridge Research Lab Tech Report 98-10, 1998. 